\title{
Exponential Stability of Laminated Beam with Constant Delay Feedback
}

\author{
Kassimu Mpungu ${ }^{a}$ and Tijani A. Apalara ${ }^{b}$ \\ ${ }^{a}$ Universiti Teknologi Malaysia \\ 81310 Johor Bahru, Johor, Malaysia \\ ${ }^{b}$ University of Hafr Al Batin \\ 31991 Hafr Al-Batin, Saudi Arabia \\ E-mail(corresp.): mpungu@graduate.utm.my \\ E-mail: tijani@uhb.edu.sa
}

Received October 19, 2020; revised September 14, 2021; accepted September 14, 2021

\begin{abstract}
In this article, we consider a system of laminated beams with an internal constant delay term in the transverse displacement. We prove that the dissipation through structural damping at the interface is strong enough to exponentially stabilize the system under suitable assumptions on delay feedback and coefficients of wave propagation speed.
\end{abstract}

Keywords: laminated beam, interfacial slip, constant delay, exponential decay.

AMS Subject Classification: 35L56; 93D15; 93D23.

\section{Introduction}

In this work, we are concerned with a model governing vibrations in a structure made up of two layered beams popularly known as "Laminated beam model", subjected to an internal constant delay term acting on the transverse displacement. Derived by Hansen et al. [15], the laminated beam model describes the vibrations in a structure consisting of two layered identical beams of uniform thickness stuck together by an adhesive (of negligible thickness), in such a way that a slip is permitted while they are continuously in contact with each other. In the absence of interfering forces, the system of the model takes the following

Copyright (c) 2021 The Author(s). Published by Vilnius Gediminas Technical University This is an Open Access article distributed under the terms of the Creative Commons Attribution License (http://creativecommons.org/licenses/by/4.0/), which permits unrestricted use, distribution, and reproduction in any medium, provided the original author and source are credited. 
form

$$
\left\{\begin{array}{l}
\rho w_{t t}+G\left(\psi-w_{x}\right)_{x}=0 \\
I_{\rho}\left(3 s_{t t}-\psi_{t t}\right)-D\left(3 s_{x x}-\psi_{x x}\right)-G\left(\psi-w_{x}\right)=0 \\
3 I_{\rho} s_{t t}-3 D s_{x x}+3 G\left(\psi-w_{x}\right)+4 \gamma s+4 \beta s_{t}=0
\end{array}\right.
$$

with $x \in(0,1)$ and $t \geq 0$. Here $\rho, I_{\rho}, G, D, \beta$, and, $\gamma$ are density, mass moment of inertia, shear stiffness, flexural rigidity, adhesive damping parameter and, adhesive stiffness respectively. Similarly, $w=w(x, t)$ denotes the transverse displacement of the beam from its equilibrium position, $\psi=\psi(x, t)$ is the rotation angle, $3 s-\psi$ denotes the effective rotation angle and, $s=s(x, t)$ is proportional to the amount of slip along the interface. The first two equations of (1.1) are derived on the assumption of Timoshenko beam theory and, the third equation describes the dynamics of the slip. Moreover, if $s(x, t)$ is identically zero, then the standard Timoshenko system is recovered. Furthermore, if $\beta \neq 0$, then the adhesion at the interface produces a restoration comparable force to counteract the interfacial slip. Otherwise, in absence of adhesive damping (i.e. $\beta=0$ ), the third equation of describes the dynamics of slip of coupled laminated beam without structural damping.

Laminated beams have wider applications in engineering as structures are often made out of more than one beam or plate stuck together using the appropriate substance depending on their intended purposes. Among other applications, the closest examples one can think of in recent times are the layered glass gorilla screen protection for smart gadgets, windscreens, among others. Being a controlled system, stability is very important. Thus, many researchers among mathematicians and engineers have focused a lot of attention on the study of well-posedness and more importantly, the stability behavior of this differential model, majorly by exploiting different damping mechanisms introduced to the system. We discus some of the results below.

The asymptotic behavior of system (1.1) with boundary feedback controls of the form

$$
\left\{\begin{array}{l}
w(0, t)=\psi(0, t)=s(0, t), \quad\left(\psi-w_{x}\right)(1, t)=k_{1} w_{t}(1, t), \\
\left(3 s_{x}-\psi_{x}\right)(1, t)=-k_{2}\left(3 s_{t}-\psi_{t}\right)(1, t)
\end{array}\right.
$$

was studied by Wang et al. [32]. The authors established an exponential stability of the system provided that $r_{1}=\sqrt{\frac{\rho}{G}} \neq \sqrt{\frac{I_{\rho}}{D}}=r_{2}, \quad k_{i} \neq r_{i}(i=1,2)$. Interestingly, Tatar [31] and Mustafa [24] obtained the result in [32] under weaker conditions on the parameters $\rho, G, I_{\rho}$, and $D$. Some related results were also obtained by Cao et al [10] with different boundary controls.

Apart from stabilization through boundary damping mechanisms, researchers have considered other interesting damping techniques. For example, Raposo [28] introduced extra linear frictional damping terms in the first two equations of (1.1) in addition to structural damping, and proved exponential stability without further restrictions. Later Apalara et al. [9] established that a single linear frictional damping in the effective rotation angle is sufficient for exponential decay in case of equal wave speeds. Similarly, in [2], the authors consider system (1.1) with structural damping, and prove that if it is coupled 
with boundary feedback controls acting through complementary displacements, then no further dissipation or restrictions on parameters are required for exponential decay, otherwise the assumption of equal wave speeds is necessary.

Regarding dissipation through material damping, for laminated beam with infinity memory, we mention the work in [18], in which with only structural damping and suitable assumptions on the relaxation function, the authors established general exponential decay results in case of equal wave speeds and polynomial stability otherwise. For earlier results concerning stabilization of laminated beam through viscoelastic damping, we refer the reader to $[11,21,25]$. Furthermore, regarding stabilization through thermal effects, we cite the result in [20]. The authors investigated a thermoelastic laminated beam with past history, and proved that in presence of structural damping, the solution decays exponentially and polynomially without any restrictions on the parameters. For a system without structural damping, exponential and polynomial decay of the solution are possible in case of equal wave speeds, otherwise, the system lacks exponential stability. Other interesting results about damping through thermal effects can be found in $[4,5,14]$ for thermoelasticity, and [19] for thermoelasticity of type III. In all these works, authors mainly established that the system decays exponentially in the case of equal wave speeds and polynomially otherwise, with and without structural damping.

In control systems, time delays are inherent since propagation and transport of material and/or information are involved. Time delay may manifest in form of lags between the input and processing the output, or lags in attaining or restoring the desired system stability after perturbations due to internal or external factors, among others. To explicitly analyse the delay effect on physical properties especially stability, it is preferred that control systems are modeled and represented by delay differential equations. Although there are isolated cases where that voluntary inclusion of delay may benefit control (see [1]) or may not significantly disturb the general system stability, for instance, in [23], time lags have been established as one of the underlying causes of instability and deterioration of the system performance. For example, consider the following system of wave equation

$$
\left\{\begin{array}{lr}
\varphi_{t t}-\Delta \varphi=0, & \text { in } \Omega \times(0, \infty), \\
\varphi=0, & \text { on } \Gamma_{0} \times(0, \infty), \\
\frac{\partial \varphi}{\partial \nu}=-\mu_{1} \varphi_{t}-\mu_{2} \varphi_{t}(x, t-\tau), & \text { on } \Gamma_{1} \times(0, \infty),
\end{array}\right.
$$

where $\varphi=\varphi(x, t), \quad \Omega \subset \mathbb{R}^{2}$ is open and bounded having a smooth boundary $\partial \Omega \equiv \Gamma_{0} \cup \Gamma_{1}$ and, $\nu=\nu(x)$ is the unit normal to $\partial \Omega$. It is long established that in the absence of delay $\left(\mu_{2}=0, \mu_{1}>0\right)$, the system (1.2) is exponentially stable, see $[16,17,34]$.Whereas, on including delay $\left(\mu_{2}>0\right)$, Nicaise et al. [26] established that the solution decays exponentially provided that $\mu_{2}<\mu_{1}$, and in case of a reversed scenario $\left(\mu_{2} \geq \mu_{1}\right)$, the authors proved that the system solutions become chaotic by introducing a correlating sequence of delays to the solution. Similar conclusions were reached by $[12,33]$. For more works regarding constant time delay effect on stability, we refer the reader to $[3,7,27,29]$ and references therein. 
In the dynamic Timoshenko beam model, the amplitude of vibrations of the complementary displacements vanishes due to damping. A constant time delay translates into a forward phase shift increasing early time response, which is seen to cause frequency dispersion in displacements [22]. This may require stronger damping to counteract the longer time needed for decay. This delay effect is inherent in the laminated beam model as it is derived on assumption of Timoshenko beam theory. The presence of structural damping in a laminated beam provides some dissipation, which is sufficient for exponential stability in absence of delay on assumption of equal wave speeds $[6,8]$. It is yet to be established if the internal structural damping can still solely stabilize the system in presence of delay, rather authors have chosen other damping mechanisms. For instance, Feng [13], considered a laminated beam with three internal constant delay feedbacks, with help of three external boundary controls and some conditions on the system parameters, he established exponential decay result. Seghour et al. [30] on the other hand, investigated a thermoelastic laminated beam with neutral delay in dynamics of slip equation, and established uniform stability provided $\rho=G I_{\rho}$. The required dissipation was obtained through thermal effects in addition to linear frictional damping in the transverse displacement.

Considering all the above observations, a natural question arises. Is exponential decay achievable for a classical laminated beam system with constant delay without additional internal damping mechanisms or dissipation through boundary controls? If yes, under what conditions? Our concern is to answer this question affirmatively. Precisely, we consider a system of laminated beam with constant delay term acting on the transverse displacement:

$$
\left\{\begin{array}{lr}
\rho w_{t t}+G\left(\psi-w_{x}\right)_{x}+\mu w_{t}(x, t-\tau)=0, & \text { in }(0,1) \times(0, \infty), \\
I_{\rho}\left(3 s_{t t}-\psi_{t t}\right)-D\left(3 s_{x x}-\psi_{x x}\right)-G\left(\psi-w_{x}\right)=0, & \text { in }(0,1) \times(0, \infty), \\
3 I_{\rho} s_{t t}-3 D s_{x x}+3 G\left(\psi-w_{x}\right)+4 \gamma s+4 \beta s_{t}=0, & \text { in }(0,1) \times(0, \infty), \\
w_{t}(x,-t)=f_{0}(x, t), & \text { in }(0,1) \times(0, \tau), \\
w(x, 0)=w_{0}, s(x, 0)=s_{0}, \psi(x, 0)=\psi_{0}, & \text { in }(0,1), \\
w_{t}(x, 0)=w_{1}, s_{t}(x, 0)=s_{1}, \psi_{t}(x, 0)=\psi_{1}, & \text { in }(0,1), \\
w(0, t)=s_{x}(0, t)=\psi_{x}(0, t)=0, & \text { in }(0, \infty), \\
w_{x}(1, t)=s(1, t)=\psi(1, t)=0, & \text { in }(0, \infty),
\end{array}\right.
$$

where, $w_{0}, w_{1}, \psi_{0}, \psi_{1}, s_{0}, s_{1}, f_{0}$ is the initial data which belongs to an appropriate space, $\tau>0$ is time delay and the none zero real number $\mu$ is the weight of delay. With some restrictions on $\mu$, we prove that the adhesive damping is strong enough to stabilize the system exponentially, even in presence of delay without any other additional damping or boundary controls, provided the assumption of equal wave propagation speed $\left(G I_{\rho}=\rho D\right)$ holds.

The rest of the paper is organized as follows. We present some preliminaries in Section 2. In Section 3, we state and prove some technical lemmas and finally in Section 4, we discuss the stability result. 


\section{Preliminaries}

We proceed by introducing the following new variable as in [26].

$$
z(x, \sigma, t)=w_{t}(x, t-\tau \sigma) \quad \text { in }(0,1) \times(0,1) \times(0, \infty) .
$$

It follows directly that $z$ satisfies

$$
\tau z_{t}(x, \sigma, t)+z_{\sigma}(x, \sigma, t)=0 \quad \text { in }(0,1) \times(0,1) \times(0, \infty) .
$$

Consequently, the system (1.3) is equivalent to

$$
\left\{\begin{array}{l}
\rho w_{t t}+G\left(\psi-w_{x}\right)_{x}+\mu z(x, 1, t)=0, \quad \text { in }(0,1) \times(0, \infty), \\
I_{\rho}\left(3 s_{t t}-\psi_{t t}\right)-D\left(3 s_{x x}-\psi_{x x}\right)-G\left(\psi-w_{x}\right)=0, \text { in }(0,1) \times(0, \infty), \\
3 I_{\rho} s_{t t}-3 D s_{x x}+3 G\left(\psi-w_{x}\right)+4 \gamma s+4 \beta s_{t}=0, \quad \text { in }(0,1) \times(0, \infty), \\
\tau z_{t}(x, \sigma, t)+z_{\sigma}(x, \sigma, t)=0, \quad \text { in }(0,1) \times(0,1) \times(0, \infty), \\
z(x, 0, t)=w_{t}(x, t), \quad \text { in }(0,1) \times(0, \infty), \\
z(x, \sigma, 0)=f_{0}(x, \tau \sigma), \quad \operatorname{in}(0,1) \times(0,1), \\
w(x, 0)=w_{0}, s(x, 0)=s_{0}, \psi(x, 0)=\psi_{0}, \quad \text { in }(0,1), \\
w_{t}(x, 0)=w_{1}, s_{t}(x, 0)=s_{1}, \psi_{t}(x, 0)=\psi_{1}, \quad \text { in }(0,1), \\
w(0, t)=s_{x}(0, t)=\psi_{x}(0, t)=0, \quad \text { in }(0, \infty), \\
w_{x}(1, t)=s(1, t)=\psi(1, t)=0, \quad \text { in }(0, \infty) .
\end{array}\right.
$$

The energy of the solution to the system (2.1) is given by

$$
\begin{aligned}
E(t)= & \frac{1}{2} \int_{0}^{1}\left[\rho w_{t}^{2}+I_{\rho}\left(3 s_{t}-\psi_{t}\right)^{2}+D\left(3 s_{x}-\psi_{x}\right)^{2}+3 I_{\rho} s_{t}^{2}+3 D s_{x}^{2}\right] d x \\
& +\frac{1}{2} \int_{0}^{1}\left[4 \gamma s^{2}+G\left(\psi-w_{x}\right)^{2}+\tau|\mu| \int_{0}^{1} z^{2}(x, \sigma) d \sigma\right] d x .
\end{aligned}
$$

On the existence, uniqueness, and smoothness of solution of problem (2.1), we introduce the vector function $\Phi=(w, u, \xi, v, s, z)^{T} ; u=w_{t}, \xi=3 s-\psi, v=$ $\xi_{t}$, and $y=s_{t}$, and thereby transform system (2.1) to

$$
\left\{\begin{array}{l}
\frac{d}{d t} \Phi(t)=\mathcal{A} \Phi(t), \quad t>0 \\
\Phi(0)=\Phi_{0}=\left(w_{0}, w_{1}, 3 s_{0}-\psi_{0}, 3 s_{1}-\psi_{1}, s_{0}, s_{1}, f_{0}\right)^{T},
\end{array}\right.
$$

where the operator $\mathcal{A}$ is defined by

$$
\mathcal{A} \Phi=\left(\begin{array}{c}
u \\
-\frac{1}{\rho}\left(G\left(3 s-\xi-w_{x}\right)_{x}+\mu z(x, 1)\right) \\
v \\
\frac{1}{I_{\rho}}\left(D \xi_{x x}+G\left(3 s-\xi-w_{x}\right)\right) \\
y \\
\frac{1}{I_{\rho}}\left(D s_{x x}-G\left(3 s-\xi-w_{x}\right)-\frac{4 \gamma}{3} s-\frac{4 \beta}{3} y\right) \\
-\frac{1}{\tau} z_{\sigma}(x, \sigma)
\end{array}\right) .
$$


We consider the following spaces

$$
H_{a}^{1}=\left\{v: v \in H^{1}(0,1): v(0)=0\right\}, \quad H_{b}^{1}=\left\{v: v \in H^{1}(0,1): v(1)=0\right\}
$$

and let

$$
\begin{aligned}
\mathcal{H}:= & H_{a}^{1}(0,1) \times L^{2}(0,1) \times H_{b}^{1}(0,1) \times L^{2}(0,1) \times H_{b}^{1}(0,1) \times L^{2}(0,1) \\
& \times L^{2}((0,1) \times(0,1))
\end{aligned}
$$

be the Hilbert space equipped with the following inner product

$$
\begin{aligned}
(\Phi, \tilde{\Phi})_{\mathcal{H}}= & \rho \int_{0}^{1} u \tilde{u} d x+G \int_{0}^{1}\left(3 s-\xi-w_{x}\right)\left(3 \tilde{s}-\tilde{\xi}-\tilde{w}_{x}\right) d x+I_{\rho} \int_{0}^{1} v \tilde{v} d x \\
& +3 I_{\rho} \int_{0}^{1} y \tilde{y} d x+D \int_{0}^{1} \xi_{x} \tilde{\xi}_{x} d x+4 \gamma \int_{0}^{1} s \tilde{s} d x+3 D \int_{0}^{1} s_{x} \tilde{s}_{x} d x \\
& +\tau|\mu| \int_{0}^{1} \int_{0}^{1} z(x, \sigma) \tilde{z}(x, \sigma) d \sigma d x .
\end{aligned}
$$

The domain of $\mathcal{A}$ is given by

$$
D(\mathcal{A})=\left\{\begin{array}{l}
\Phi \in \mathcal{H} \mid w \in H^{2}(0,1) \cap H_{a}^{1}(0,1), \quad \xi, s \in H^{2}(0,1) \cap H_{b}^{1}(0,1), \\
u \in H_{a}^{1}(0,1), \quad v, y \in H_{b}^{1}(0,1), \quad z, z_{\sigma} \in L^{2}((0,1) \times(0,1)), \\
w_{x}(1)=\xi_{x}(0)=s_{x}(0)=0
\end{array}\right\} .
$$

We observe that $D(\mathcal{A})$ is independent of time $t>0$. Furthermore, it is obvious that $D(\mathcal{A})$ is dense in $\mathcal{H}$. We have the following well-posedness result.

Theorem 1. Let $\Phi_{0} \in \mathcal{H}$, then there exists a unique weak solution $\Phi \in C\left(\mathbb{R}^{+}, \mathcal{H}\right)$ of problem (2.3). Moreover, if $\Phi_{0} \in D(\mathcal{A})$, then $\Phi \in C\left(\mathbb{R}^{+}, D(\mathcal{A})\right) \cap C^{1}\left(\mathbb{R}^{+}, \mathcal{H}\right)$.

Remark 1. Theorem 1 can be proved using the standard semigroup method as established in $[3,4,13]$.

\section{Technical lemmas}

In this section, we state and prove some technical lemmas which are fundamental in the proof of our stability result. We use multiplier technique to establish stability results for the energy of the solution of system (2.1). This requires constructing a suitable Lyapunov functional equivalent to energy as we elaborate in the subsequent section.

Lemma 1. If $(w, \psi, s, z)$ is a solution of (2.1), then the energy functional $E$, defined by (2.2) satisfies

$$
E^{\prime}(t) \leq-4 \beta \int_{0}^{1} s_{t}^{2} d x+|\mu| \int_{0}^{1} w_{t}^{2} d x, \quad \forall t \geq 0 .
$$


Proof. Multiplying the first three equations in the system $(2.1)$ by $w_{t},\left(3 s_{t}-\right.$ $\left.\psi_{t}\right)$ and $s_{t}$ respectively, then integrating each by parts over $(0,1)$ using the boundary conditions, we end up with

$$
\begin{aligned}
& \frac{1}{2} \frac{d}{d t} \int_{0}^{1}\left[\rho w_{t}^{2}+I_{\rho}\left(3 s_{t}-\psi_{t}\right)^{2}+D\left(3 s_{x}-\psi_{x}\right)^{2}+3 I_{\rho} s_{t}^{2}+3 D s_{x}^{2}+4 \gamma s^{2}\right] d x \\
& \quad+\frac{1}{2} \frac{d}{d t} \int_{0}^{1}\left[G\left(\psi-w_{x}\right)^{2}\right] d x=-\mu \int_{0}^{1} z(x, 1) w_{t} d x-4 \beta \int_{0}^{1} s_{t}^{2} d x .
\end{aligned}
$$

Similarly, multiplying $(2.1)_{4}$ by $|\mu| z$, followed by integrating the product over $(0,1) \times(0,1)$ and then using the substitution $z(x, 0, t)=w_{t}$, we obtain

$$
\frac{\tau|\mu|}{2} \frac{d}{d t} \int_{0}^{1} \int_{0}^{1} z^{2}(x, \sigma) d \sigma d x=-\frac{|\mu|}{2} \int_{0}^{1} z^{2}(x, 1) d x+\frac{|\mu|}{2} \int_{0}^{1} w_{t}^{2} d x .
$$

Next, merging (3.2) and (3.3), we note that from (2.2),

$$
E^{\prime}(t)=-\mu \int_{0}^{1} z(x, 1) w_{t} d x-4 \beta \int_{0}^{1} s_{t}^{2} d x-\frac{|\mu|}{2} \int_{0}^{1} z^{2}(x, 1) d x+\frac{|\mu|}{2} \int_{0}^{1} w_{t}^{2} d x .
$$

We now exploit Young's inequality on the first term of (3.4) to obtain

$$
-\mu \int_{0}^{1} z(x, 1) w_{t} d x \leq \frac{|\mu|}{2} \int_{0}^{1} z^{2}(x, 1) d x+\frac{|\mu|}{2} \int_{0}^{1} w_{t}^{2} d x .
$$

Consequently, substituting (3.5) in (3.4) completes the proof of (3.1).

Lemma 2. If $(w, \psi, s, z)$ is a solution of (2.1), then the functional $F_{1}$, defined by

$$
F_{1}(t):=-\rho \int_{0}^{1} w w_{t} d x+\rho \int_{0}^{1} w_{t} \int_{0}^{x} \psi(y) d y d x
$$

for any $\varepsilon_{1}>0$, satisfies the estimate

$$
\begin{aligned}
\frac{d}{d t} F_{1}(t) \leq & -\frac{\rho}{2} \int_{0}^{1} w_{t}^{2} d x+\rho \int_{0}^{1}\left(3 s_{t}-\psi_{t}\right)^{2} d x+\varepsilon_{1} \int_{0}^{1} z^{2}(x, 1) d x \\
& +9 \rho \int_{0}^{1} s_{t}^{2} d x+\left(\frac{G}{2}+\frac{\mu^{2}}{4 \varepsilon_{1}}\right) \int_{0}^{1}\left(\psi-w_{x}\right)^{2} d x
\end{aligned}
$$

Proof. Differentiating $F_{1}$, using the first equation in (2.1), integrating by parts the term containing $\left(\psi-w_{x}\right)_{x}$ and exploiting the fact that $\psi_{t}=-\left(3 s_{t}-\psi_{t}\right)+$ $3 s_{t}$, we deduce that

$$
\begin{gathered}
\frac{d}{d t} F_{1}(t)=-\rho \int_{0}^{1} w_{t}^{2} d x+G \int_{0}^{1}\left(\psi-w_{x}\right)^{2} d x-\rho \int_{0}^{1} w_{t} \int_{0}^{x}\left(3 s_{t}-\psi_{t}\right)(y) d y d x \\
+3 \rho \int_{0}^{1} w_{t} \int_{0}^{x} s_{t}(y) d y d x-\mu \int_{0}^{1}\left(\int_{0}^{x} \psi(y) d y-w\right) z(x, 1) d x .
\end{gathered}
$$


By Young's and Poincaré's inequalities, the last three terms of (3.7) give

$$
\begin{aligned}
&-\rho \int_{0}^{1} w_{t} \int_{0}^{x}\left(3 s_{t}-\psi_{t}\right)(y) d y d x \leq \rho \int_{0}^{1}\left(\int_{0}^{x}\left(3 s_{t}-\psi_{t}\right) d y\right)^{2} d x+\frac{\rho}{4} \int_{0}^{1} w_{t}^{2} d x \\
& \leq \rho \int_{0}^{1}\left(3 s_{t}-\psi_{t}\right)^{2} d x+\frac{\rho}{4} \int_{0}^{1} w_{t}^{2} d x \\
& 3 \rho \int_{0}^{1} w_{t} \int_{0}^{x} s_{t}(y) d y \leq 9 \rho \int_{0}^{1}\left(\int_{0}^{x} s_{t}(y) d y\right)^{2} d x+\frac{\rho}{4} \int_{0}^{1} w_{t}^{2} d x \\
& \leq 9 \rho \int_{0}^{1} s_{t}^{2} d x+\frac{\rho}{4} \int_{0}^{1} w_{t}^{2} d x
\end{aligned}
$$

and,

$$
\begin{aligned}
& -\mu \int_{0}^{1}\left(\int_{0}^{x} \psi(y) d y-w\right) z(x, 1) d x \leq \frac{\mu^{2}}{4 \varepsilon_{1}} \int_{0}^{1}\left(\int_{0}^{x} \psi(y) d y-w\right)^{2} d x \\
& +\varepsilon_{1} \int_{0}^{1} z^{2}(x, 1) d x \leq \frac{\mu^{2}}{4 \varepsilon_{1}} \int_{0}^{1}\left(\psi-w_{x}\right)^{2} d x+\varepsilon_{1} \int_{0}^{1} z^{2}(x, 1) d x .
\end{aligned}
$$

The combination of $(3.7)-(3.8)$ leads to (3.6).

Lemma 3. If $(w, \psi, s, z)$ is a solution of (2.1), then the functional $F_{2}$, defined by

$$
F_{2}(t):=-I_{\rho} \int_{0}^{1}\left(3 s_{t}-\psi_{t}\right)(3 s-\psi) d x
$$

satisfies the estimate

$$
\begin{aligned}
\frac{d}{d t} F_{2}(t) \leq & -I_{\rho} \int_{0}^{1}\left(3 s_{t}-\psi_{t}\right)^{2} d x+\frac{3 D}{2} \int_{0}^{1}\left(3 s_{x}-\psi_{x}\right)^{2} d x \\
& +\frac{G^{2}}{2 D} \int_{0}^{1}\left(\psi-w_{x}\right)^{2} d x
\end{aligned}
$$

Proof. By direct computations using the second equation in (2.1), we obtain

$$
\begin{aligned}
\frac{d}{d t} F_{2}(t)= & -I_{\rho} \int_{0}^{1}\left(3 s_{t}-\psi_{t}\right)^{2} d x+D \int_{0}^{1}\left(3 s_{x}-\psi_{x}\right)^{2} d x \\
& -G \int_{0}^{1}\left(\psi-w_{x}\right)(3 s-\psi) d x .
\end{aligned}
$$

Exploiting Young's and Poincaré's inequalities, we estimate the last term of (3.10) as follows:

$$
\begin{aligned}
& -G \int_{0}^{1}\left(\psi-w_{x}\right)(3 s-\psi) d x \leq \frac{G^{2}}{2 D} \int_{0}^{1}\left(\psi-w_{x}\right)^{2} d x+\frac{D}{2} \int_{0}^{1}(3 s-\psi)^{2} d x \\
& \quad \leq \frac{G^{2}}{2 D} \int_{0}^{1}\left(\psi-w_{x}\right)^{2} d x+\frac{D}{2} \int_{0}^{1}\left(3 s_{x}-\psi_{x}\right)^{2} d x
\end{aligned}
$$

Consequently, the relation (3.9) follows directly by substituting (3.11) into (3.10). 
Lemma 4. If $(w, \psi, s, z)$ is a solution of (2.1), then the functional $F_{3}$, defined by

$$
F_{3}(t):=3 I_{\rho} \int_{0}^{1} s_{t} s d x+2 \beta \int_{0}^{1} s^{2} d x+3 \rho \int_{0}^{1} w_{t} \int_{0}^{x} s(y) d y d x
$$

for any $\varepsilon_{2}>0$, satisfies the estimate:

$$
\begin{aligned}
\frac{d}{d t} F_{3}(t) & \leq-3 D \int_{0}^{1} s_{x}^{2} d x-3 \gamma \int_{0}^{1} s^{2} d x+\varepsilon_{2} \int_{0}^{1} w_{t}^{2} d x+\frac{9 \mu^{2}}{4 \gamma} \int_{0}^{1} z^{2}(x, 1) d x \\
+ & \left(3 I_{\rho}+\frac{9 \rho^{2}}{4 \varepsilon_{2}}\right) \int_{0}^{1} s_{t}^{2} d x
\end{aligned}
$$

Proof. Differentiating $F_{3}$, using (2.1), and then integrating by part the terms containing $\left(\psi-w_{x}\right)_{x}$ and $s_{x x}$, we arrive at

$$
\begin{aligned}
\frac{d}{d t} F_{3}(t)= & -3 D \int_{0}^{1} s_{x}^{2} d x-4 \gamma \int_{0}^{1} s^{2} d x+3 \rho \int_{0}^{1} w_{t} \int_{0}^{x} s_{t}(y) d y d x \\
& +3 I_{\rho} \int_{0}^{1} s_{t}^{2} d x-3 \mu \int_{0}^{1} z(x, 1) \int_{0}^{x} s(y) d y d x
\end{aligned}
$$

Using Young's, Poincaré's and Cauchy-Schwarz inequalities, we have

$$
\begin{aligned}
& -3 \mu \int_{0}^{1} z(x, 1) \int_{0}^{x} s(y) d y d x \leq \frac{9 \mu^{2}}{4 \gamma} \int_{0}^{1} z^{2}(x, 1) d x+\gamma \int_{0}^{1}\left(\int_{0}^{x} s(y) d y\right)^{2} d x \\
& \quad \leq \frac{9 \mu^{2}}{4 \gamma} \int_{0}^{1} z^{2}(x, 1) d x+\gamma \int_{0}^{1} s^{2} d x \\
& 3 \rho \int_{0}^{1} w_{t} \int_{0}^{x} s_{t}(y) d y d x \leq \varepsilon_{2} \int_{0}^{1} w_{t}^{2} d x+\frac{9 \rho^{2}}{4 \varepsilon_{2}} \int_{0}^{1}\left(\int_{0}^{x} s_{t}(y) d y\right)^{2} d x \\
& \quad \leq \varepsilon_{2} \int_{0}^{1} w_{t}^{2} d x+\frac{9 \rho^{2}}{4 \varepsilon_{2}} \int_{0}^{1} s_{t}^{2} d x
\end{aligned}
$$

for any $\varepsilon_{2}>0$. Estimate (3.12) follows directly by virtue of $(3.13)-(3.15)$.

The assumption of equal wave speeds $G I_{\rho}=\rho D$ plays an important role in the next two lemmas.

Lemma 5. If $(w, \psi, s, z)$ is a solution of (2.1), then the functional $F_{4}$, defined by

$$
F_{4}(t):=-\int_{0}^{1}\left(3 s_{t}-\psi_{t}\right) w_{x} d x-\int_{0}^{1}\left(3 s_{x}-\psi_{x}\right) w_{t} d x+3 \int_{0}^{1}\left(3 s_{t}-\psi_{t}\right) s d x
$$

for any $\varepsilon_{3}>0$, satisfies the estimate

$$
\begin{gathered}
\frac{d}{d t} F_{4}(t) \leq-\frac{D}{2 I_{\rho}} \int_{0}^{1}\left(3 s_{x}-\psi_{x}\right)^{2} d x+\varepsilon_{3} \int_{0}^{1}\left(3 s_{t}-\psi_{t}\right)^{2} d x+\frac{9}{\varepsilon_{3}} \int_{0}^{1} s_{t}^{2} d x \\
+\frac{I_{\rho} \mu^{2}}{D \rho^{2}} \int_{0}^{1} z^{2}(x, 1) d x+\left(\frac{G}{I_{\rho}}+\frac{G^{2}}{D I_{\rho}}\right) \int_{0}^{1}\left(\psi-w_{x}\right)^{2} d x
\end{gathered}
$$


Proof. As in the previous Lemmas, direction computations using (2.1), integration by parts the term containing $3 s_{x x}-\psi_{x x}$, and exploiting the fact that $w_{x}=-\left(\psi-w_{x}\right)-(3 s-\psi)+3 s$, we end up with

$$
\begin{gathered}
\frac{d}{d t} F_{4}(t)=-\frac{D}{I_{\rho}} \int_{0}^{1}\left(3 s_{x}-\psi_{x}\right)^{2} d x+\frac{G}{I_{\rho}} \int_{0}^{1}\left(\psi-w_{x}\right)^{2} d x+3 \int_{0}^{1}\left(3 s_{t}-\psi_{t}\right) s_{t} d x \\
+\frac{G}{I_{\rho}} \int_{0}^{1}\left(\psi-w_{x}\right)(3 s-\psi) d x+\frac{\mu}{\rho} \int_{0}^{1}\left(3 s_{x}-\psi_{x}\right) z(x, 1) d x
\end{gathered}
$$

Next, Young's and Poincaré's inequalities guarantee the relations

$$
\begin{aligned}
3 \int_{0}^{1}\left(3 s_{t}-\psi_{t}\right) s_{t} d x & \leq \varepsilon_{3} \int_{0}^{1}\left(3 s_{t}-\psi_{t}\right)^{2} d x+\frac{9}{\varepsilon_{3}} \int_{0}^{1} s_{t}^{2} d x \\
\frac{G}{I_{\rho}} \int_{0}^{1}\left(\psi-w_{x}\right)(3 s-\psi) d x & \leq \frac{G^{2}}{D I_{\rho}} \int_{0}^{1}\left(\psi-w_{x}\right)^{2} d x+\frac{D}{4 I_{\rho}} \int_{0}^{1}(3 s-\psi)^{2} d x \\
& \leq \frac{G^{2}}{D I_{\rho}} \int_{0}^{1}\left(\psi-w_{x}\right)^{2} d x+\frac{D}{4 I_{\rho}} \int_{0}^{1}\left(3 s_{x}-\psi_{x}\right)^{2} d x
\end{aligned}
$$

and,

$$
\frac{\mu}{\rho} \int_{0}^{1}\left(3 s_{x}-\psi_{x}\right) z(x, 1) d x \leq \frac{D}{4 I_{\rho}} \int_{0}^{1}\left(3 s_{x}-\psi_{x}\right)^{2} d x+\frac{I_{\rho} \mu^{2}}{D \rho^{2}} \int_{0}^{1} z^{2}(x, 1) d x,
$$

for any $\varepsilon_{3}>0$. Estimate (3.16) follows directly by substituting (3.18)-(3.19) into (3.17).

Lemma 6. If $(w, \psi, s, z)$ is a solution of (2.1), then the functional $F_{5}$, defined by

$$
F_{5}(t):=\int_{0}^{1}\left(\psi-w_{x}\right) s_{t} d x-\int_{0}^{1} w_{t} s_{x} d x
$$

for any $\varepsilon_{4}, \varepsilon_{5}>0$, satisfies the estimate

$$
\begin{aligned}
\frac{d}{d t} F_{5}(t) & \leq-\frac{G}{2 I_{\rho}} \int_{0}^{1}\left(\psi-w_{x}\right)^{2} d x+\varepsilon_{4} \int_{0}^{1}\left(3 s_{t}-\psi_{t}\right)^{2} d x+\varepsilon_{5} \int_{0}^{1} z^{2}(x, 1) d x \\
+ & \left(\frac{16 \gamma^{2}}{9 I_{\rho}}+\frac{\mu^{2}}{4 \rho^{2} \varepsilon_{5}}\right) \int_{0}^{1} s_{x}^{2} d x+\left(3+\frac{1}{4 \varepsilon_{4}}+\frac{16 \beta^{2}}{9 I_{\rho}}\right) \int_{0}^{1} s_{t}^{2} d x .
\end{aligned}
$$

Proof. Differentiating $F_{5}$ then integrating by parts over $(0,1)$ the term containing $s_{x t}$ and, using the substitution $w_{x t}=-\left(3 s_{t}-\psi_{t}\right)-\left(\psi-w_{x}\right)_{t}+3 s_{t}$, we arrive at

$$
\frac{d}{d t} F_{5}(t)=\int_{0}^{1}\left(\psi-w_{x}\right) s_{t t} d x-\int_{0}^{1} w_{t t} s_{x} d x-\int_{0}^{1}\left(3 s_{t}-\psi_{t}\right) s_{t} d x+3 \int_{0}^{1} s_{t}^{2} d x
$$


Next, using (3.21), (2.1) and ingratiating by parts the term containing $s_{x}$, we arrive at

$$
\begin{gathered}
\frac{d}{d t} F_{5}(t)=-\frac{G}{I_{\rho}} \int_{0}^{1}\left(\psi-w_{x}\right)^{2} d x+3 \int_{0}^{1} s_{t}^{2} d x-\frac{4 \gamma}{3 I_{\rho}} \int_{0}^{1} s\left(\psi-w_{x}\right) d x \\
-\frac{4 \beta}{3 I_{\rho}} \int_{0}^{1} s_{t}\left(\psi-w_{x}\right) d x-\int_{0}^{1}\left(3 s_{t}-\psi_{t}\right) s_{t} d x+\frac{\mu}{\rho} \int_{0}^{1} z(x, 1) s_{x} d x
\end{gathered}
$$

Exploiting Youngs and Poincaré's inequalities, the last four terms of (3.22) are estimated as follows

$$
\begin{aligned}
& -\frac{4 \gamma}{3 I_{\rho}} \int_{0}^{1} s\left(\psi-w_{x}\right) d x \leq \frac{G}{4 I_{\rho}} \int_{0}^{1}\left(\psi-w_{x}\right)^{2} d x+\frac{16 \gamma^{2}}{9 I_{\rho}} \int_{0}^{1} s^{2} d x \\
& \quad \leq \frac{G}{4 I_{\rho}} \int_{0}^{1}\left(\psi-w_{x}\right)^{2} d x+\frac{16 \gamma^{2}}{9 I_{\rho}} \int_{0}^{1} s_{x}^{2} d x \\
& -\frac{4 \beta}{3 I_{\rho}} \int_{0}^{1}\left(\psi-w_{x}\right) s_{t} d x \leq \frac{G}{4 I_{\rho}} \int_{0}^{1}\left(\psi-w_{x}\right)^{2} d x+\frac{16 \beta^{2}}{9 I_{\rho}} \int_{0}^{1} s_{t}^{2} d x, \\
& -\int_{0}^{1}\left(3 s_{t}-\psi_{t}\right) s_{t} d x \leq \varepsilon_{4} \int_{0}^{1}\left(3 s_{t}-\psi_{t}\right)^{2} d x+\frac{1}{4 \varepsilon_{4}} \int_{0}^{1} s_{t}^{2} d x \\
& \frac{\mu}{\rho} \int_{0}^{1} z(x, 1) s_{x} d x \leq \frac{\mu^{2}}{4 \rho^{2} \varepsilon_{5}} \int_{0}^{1} s_{x}^{2} d x+\varepsilon_{5} \int_{0}^{1} z^{2}(x, 1) d x
\end{aligned}
$$

for any $\varepsilon_{4}, \varepsilon_{5}>0$. The assertion of the lemma follows from the estimates $(3.23)-(3.24)$ and (3.22).

Lemma 7. If $(w, \psi, s, z)$ is a solution of (2.1), then the functional $F_{6}$, defined by

$$
F_{6}(t):=\tau \int_{0}^{1} \int_{0}^{1} e^{-\sigma \tau} z^{2}(x, \sigma) d \sigma d x
$$

satisfies, for $m_{1}>0$ the estimate:

$$
\frac{d}{d t} F_{6}(t) \leq-m_{1} \int_{0}^{1} z^{2}(x, 1) d x-m_{1} \tau \int_{0}^{1} \int_{0}^{1} z^{2}(x, \sigma) d \sigma d x+\int_{0}^{1} w_{t}^{2} d x .
$$

Proof. Differentiate $F_{6}$ and use the fourth equation in $(2.1)$ and $z(x, 0)=w_{t}$ as follows

$$
\begin{aligned}
\frac{d}{d t} & F_{6}(t)=-2 \int_{0}^{1} \int_{0}^{1} e^{-\tau \sigma} z(x, \sigma) z_{\sigma}(x, \sigma) d \sigma d x \\
= & -\int_{0}^{1} \int_{0}^{1} \frac{d}{d \sigma}\left[e^{-\tau \sigma} z^{2}(x, \sigma)\right] d \sigma d x-\tau \int_{0}^{1} \int_{0}^{1} e^{-\tau \sigma} z^{2}(x, \sigma) d \sigma d x \\
& =-\int_{0}^{1}\left[e^{-\tau} z^{2}(x, 1)-z^{2}(x, 0)\right] d x-\tau \int_{0}^{1} \int_{0}^{1} e^{-\tau \sigma} z^{2}(x, \sigma) d \sigma d x \\
& =-\int_{0}^{1} e^{-\tau} z^{2}(x, 1) d x+\int_{0}^{1} w_{t}^{2} d x-\tau \int_{0}^{1} \int_{0}^{1} e^{-\tau \sigma} z^{2}(x, \sigma) d \sigma d x .
\end{aligned}
$$

Observe that, $\forall \sigma \in(0,1)$, the relation $e^{-\tau} \leq e^{-\sigma \tau} \leq 1$ holds. Therefore, for some $m_{1}=e^{-\tau}$, we arrive at the estimate (3.25). 


\section{Exponential stability}

In this section, using the lemmas obtained in Section 3, we state and prove our main stability results.

Lemma 8. Let $N, N_{k}, k=1, \ldots, 6$, be positive constants. The functional defined by

$$
\mathcal{L}(t):=N E(t)+\sum_{k=1}^{6} N_{k} F_{k}(t), \quad N_{k}>0, k=1, \ldots, 6, t \geq 0
$$

satisfies the equivalence relation

$$
c_{1} E(t) \leq \mathcal{L}(t) \leq c_{2} E(t), \quad \forall t \geq 0
$$

for some positive constants $c_{1}$ and $c_{2}$.

Proof. Let $\mathfrak{L}(t)=\sum_{k=1}^{6} N_{k} F_{k}(t)$.

$$
\begin{aligned}
|\mathfrak{L}(t)| \leq & \rho N_{1} \int_{0}^{1}\left|w w_{t}\right| d x+\rho N_{1} \int_{0}^{1}\left|w_{t} \int_{0}^{x} \psi(y) d y\right| d x \\
& +I_{\rho} N_{2} \int_{0}^{1}\left|\left(3 s_{x}-\psi_{x}\right)\left(3 s_{t}-\psi_{t}\right)\right| d x+3 I_{\rho} N_{3} \int_{0}^{1}\left|s_{t} s\right| d x \\
& +2 \beta N_{3} \int_{0}^{1} s^{2} d x+3 \rho N_{3} \int_{0}^{1}\left|w_{t} \int_{0}^{x} s(y) d y\right| d x \\
& +3 N_{4} \int_{0}^{1}\left|\left(3 s_{t}-\psi_{t}\right) s\right| d x+N_{4} \int_{0}^{1}\left|\left(3 s_{t}-\psi_{t}\right) w_{x}\right| d x \\
& +N_{4} \int_{0}^{1}\left|\left(3 s_{x}-\psi_{x}\right) w_{t}\right| d x+N_{5} \int_{0}^{1}\left|\left(\psi-w_{x}\right) s_{t}\right| d x \\
& +N_{5} \int_{0}^{1}\left|w_{t} s_{x}\right| d x+\tau N_{6} \int_{0}^{1} \int_{0}^{1} e^{-\sigma \tau} z^{2}(x, \sigma) d \sigma d x .
\end{aligned}
$$

Exploiting Young's, Poincaré's, Cauchy-Schwarz inequalities, (2.2), accompanied with the fact that $w_{x}=-\left(\psi-w_{x}\right)-(3 s-\psi)+3 s$ and $e^{-\sigma \tau} \leq 1$ for all $\sigma \in(0,1)$, we deduce that for some positive constant $\eta$,

$$
\begin{aligned}
|\mathfrak{L}(t)| \leq & \eta \int_{0}^{1}\left[w_{t}^{2}+\left(3 s_{t}-\psi_{t}\right)^{2}+\left(3 s_{x}-\psi_{x}\right)^{2}+s_{t}^{2}+s_{x}^{2}+s^{2}+\left(\psi-w_{x}\right)^{2}\right] d x \\
& +\eta \int_{0}^{1} \int_{0}^{1} z^{2}(x, \sigma) d \sigma d x \leq \eta E(t) .
\end{aligned}
$$

It is easy to observe that, from (4.1) that $|\mathcal{L}(t)-N E(t)| \leq \eta E(t)$, which is equivalent to

$$
(N-\eta) E(t) \leq \mathcal{L}(t) \leq(N+\eta) E(t),
$$

and hence the relation (4.2) follows by taking $N$ large enough. 
At this point, we're in position to prove our stability result which reads as follows.

Theorem 2. Let $(w, \psi, s, z)$ be a solution of (2.1) and suppose that $G I_{\rho}=\rho D$, there exist a positive number $\bar{\mu}$ such that if $|\mu|<\bar{\mu}$, then the energy $E(t)$ of (2.1) defined by (2.2) vanishes exponentially as $t$ approaches infinity, i.e.

$$
E(t) \leq a e^{-b t}, \quad \forall t \geq 0,
$$

for some positive constants $a$ and $b$.

Proof. We proceed by differentiating (4.1), then substitute for functionals $F_{1}$ to $F_{6}$ using estimates $(3.6),(3.9),(3.12),(3.16),(3.20)$ and (3.25) respectively. Setting

$$
N_{1}=1, \quad \varepsilon_{1}=\varepsilon_{5}=\mu^{2}, \quad \varepsilon_{2}=\frac{\rho}{4 N_{3}}, \quad \varepsilon_{3}=\frac{I_{\rho} N_{2}}{2 N_{4}}, \quad N_{6}=|\mu|,
$$

we end up with

$$
\begin{aligned}
\mathcal{L}^{\prime}(t) \leq & -\left[4 \beta N-c_{3}-c_{3} N_{3}\left(1+N_{3}\right)-\frac{c N_{4}^{2}}{N_{2}}-c_{3} N_{5}\left(1+\frac{1}{\varepsilon_{4}}\right)\right] \int_{0}^{1} s_{t}^{2} d x \\
& -3 \gamma N_{3} \int_{0}^{1} s^{2} d x-\left[\frac{I_{\rho}}{2} N_{2}-\rho-\varepsilon_{4} N_{5}\right] \int_{0}^{1}\left(3 s_{t}-\psi_{t}\right)^{2} d x \\
& -\left[\frac{D N_{4}}{2 I_{\rho}}-\frac{3 D N_{2}}{2}\right] \int_{0}^{1}\left(3 s_{x}-\psi_{x}\right)^{2} d x-\left[3 D N_{3}-c_{3} N_{5}\right] \int_{0}^{1} s_{x}^{2} d x \\
& -\left[\frac{G N_{5}}{2 I_{\rho}}-c_{3}-c_{3} N_{2}-c_{3} N_{4}\right] \int_{0}^{1}\left(\psi-w_{x}\right)^{2} d x \\
& -|\mu|\left[m_{1}-|\mu|\left(1+\frac{9 N_{3}}{4 \gamma}+\frac{I_{\rho} N_{4}}{D \rho^{2}}+N_{5}\right)\right] \int_{0}^{1} z^{2}(x, 1) d x \\
& -m_{1} \tau|\mu| \int_{0}^{1} \int_{0}^{1} z^{2}(x, \sigma) d \sigma d x-\left[\frac{\rho}{4}-|\mu|(N+1)\right] \int_{0}^{1} w_{t}^{2} d x
\end{aligned}
$$

for some $c_{3}>0$. Next, we choose $N_{2}$ large enough such that

$$
k:=\frac{I_{\rho}}{2} N_{2}-\rho>0 .
$$

Fixing $N_{2}$ permits to choose $N_{4}$ large enough such that

$$
\frac{D N_{4}}{2 I_{\rho}}-\frac{3 D N_{2}}{2}>0
$$

With $N_{2}$ and $N_{4}$ fixed, we can easily choose $N_{5}$ large enough such that

$$
\frac{G N_{5}}{2 I_{\rho}}-c_{3}-c_{3} N_{2}-c_{3} N_{4}>0 .
$$

We pick $\varepsilon_{4}$ adequately small and $N_{3}$ sufficiently large such that

$$
k-\varepsilon_{4} N_{5}>0 \text { and } 3 D N_{3}-c_{3} N_{5}>0
$$


respectively. Next, we select $N$ sufficiently large such that (4.2) remains valid and that

$$
4 \beta N-c_{3}-c_{3} N_{3}\left(1+N_{3}\right)-\frac{c N_{4}^{2}}{N_{2}}-c_{3} N_{5}\left(1+\frac{1}{\varepsilon_{4}}\right)>0 .
$$

Finally, pick

$$
\bar{\mu}=\min \left\{\begin{array}{cc}
\frac{m_{1}}{\left(1+\frac{9 N_{3}}{4 \gamma}+\frac{I_{\rho} N_{4}}{D \rho^{2}}+N_{5}\right)}, & \frac{\rho}{4(N+1)}
\end{array}\right\}
$$

to we end up with

$$
\begin{aligned}
\mathcal{L}^{\prime}(t) \leq & -\alpha \int_{0}^{1}\left[w_{t}^{2}+s_{t}^{2}+\left(3 s_{t}-\psi_{t}\right)^{2}+\left(3 s_{x}-\psi_{x}\right)^{2}+s_{x}^{2}+s^{2}\right] d x \\
& -\alpha \int_{0}^{1}\left[\left(\psi-w_{x}\right)^{2}+z^{2}(x, 1)+\int_{0}^{1} z^{2}(x, \sigma) d \sigma\right] d x
\end{aligned}
$$

for some $\alpha>0$. By the virtue of (2.2), it is clear that for some $\alpha_{0}>0$,

$$
\mathcal{L}^{\prime}(t) \leq-\alpha_{0} E(t), \quad \forall t \geq 0
$$

It then follows directly from (4.2) and (4.4) that

$$
\mathcal{L}^{\prime}(t) \leq-b \mathcal{L}(t), \quad \forall t \geq 0,
$$

where $b=\frac{\alpha_{0}}{c_{2}}$. A simple integration of $(4.5)$ over $(0, t)$ yields

$$
\mathcal{L}(t) \leq \mathcal{L}(0) e^{-b t}, \quad \forall t \geq 0 .
$$

Consequently, the assertion of the relation (4.3) follows from (4.6) and (4.2) with $a=c_{2} E(0) / c_{1}$.

\section{Acknowledgements}

The first author appreciates Universiti Teknologi Malaysia(UTM) Research Management Center (RMC) for its continuous support. Likewise, the second author expresses sincere thanks to University of Hafr Al Batin (UHB).

\section{References}

[1] C. Abdallah, P. Dorato, J. Benitez-Read and R. Byrne. Delayed positive feedback can stabilize oscillatory systems. In Proceedings of the ACC, pp. 3106-3107. IEEE, 1993.

[2] M.S. Alves and R.N. Monteiro. Exponential stability of laminated Timoshenko beams with boundary/internal controls. J. Math. Anal. Appl., 482(1):123516, 2020. https://doi.org/10.1016/j.jmaa.2019.123516.

[3] T.A. Apalara. Asymptotic behavior of weakly dissipative Timoshenko system with internal constant delay feedbacks. Appl. Anal., 95(1):187-202, 2016. https://doi.org/10.1080/00036811.2014.1000314. 
[4] T.A. Apalara. Uniform stability of a laminated beam with structural damping and second sound. Z. Angew. Math. Phys., 68(2):41, 2017. https://doi.org/10.1007/s00033-017-0784-x.

[5] T.A. Apalara. On the stability of a thermoelastic laminated beam. Acta Math. Sci., 39(6):1517-1524, 2019. https://doi.org/10.1007/s10473-019-0604-9.

[6] T.A. Apalara. Exponential stability of laminated beams with interfacial slip. Mech. Solids, 56(1):131-137, 2021.

[7] T.A. Apalara and S.A. Messaoudi. An exponential stability result of a Timoshenko system with thermoelasticity with second sound and in the presence of delay. Appl. Math. Optim., 71(3):449-472, 2015. https://doi.org/10.1007/s00245014-9266-0.

[8] T.A. Apalara, A.M. Nass and H. Al Sulaimani. On a laminated Timoshenko beam with nonlinear structural damping. Math. Comput. Appl., 25(2):35, 2020. https://doi.org/10.3390/mca25020035.

[9] T.A. Apalara, C.A. Raposo and C.A. Nonato. Exponential stability for laminated beams with a frictional damping. Arch. Math. (Basel), 114(4):471-480, 2020.

[10] X.G. Cao, D.Y. Liu and G.Q. Xu. Easy test for stability of laminated beams with structural damping and boundary feedback controls. J. Dyn. Control Syst., 13(3):313-336, 2007. https://doi.org/10.1007/s10883-007-9022-8.

[11] Z. Chen, W. Liu and D. Chen. General decay rates for a laminated beam with memory. Taiwan. J. Math., 23(5):1227-1252, 2019. https://doi.org/10.11650/tjm/181109.

[12] R. Datko, J. Lagnese and M.P. Polis. An example on the effect of time delays in boundary feedback stabilization of wave equations. SIAM J. Control Optim., 24(1):152-156, 1986. https://doi.org/10.1137/0324007.

[13] B. Feng. Well-posedness and exponential decay for laminated Timoshenko beams with time delays and boundary feedbacks. Math. Methods Appl. Sci., 41(3):11621174, 2018. https://doi.org/10.1002/mma.4655.

[14] B. Feng. On a thermoelastic laminated Timoshenko beam: Well posedness and stability. Complexity, Art. 5139419, 2020. https://doi.org/10.1155/2020/5139419.

[15] S.W. Hansen and R.D. Spies. Structural damping in laminated beams due to interfacial slip. J. Sound Vib., 204(2):183-202, 1997. https://doi.org/10.1006/jsvi.1996.0913.

[16] V. Komornik and E. Zuazua. A direct method for the boundary stabilization of the wave equation. J. Math. Pures Appl., 69(1):33-54, 1990.

[17] I. Lasiecka. Global uniform decay rates for the solutions to wave equation with nonlinear boundary conditions. Appl. Anal., 47(1-4):191-212, 1992. https://doi.org/10.1080/00036819208840140.

[18] W. Liu, X. Kong and G. Li. Asymptotic stability for a laminated beam with structural damping and infinite memory. Math. Mech. Solids, 25(10):1979-2004, 2020. https://doi.org/10.1177/1081286520917440.

[19] W. Liu, Y. Luan, Y. Liu and G. Li. Well-posedness and asymptotic stability to a laminated beam in thermoelasticity of type III. Math. Meth. Appl. Sci., 43(6):3148-3166, 2020. https://doi.org/10.1002/mma.6108. 
[20] W. Liu and W. Zhao. Stabilization of a thermoelastic laminated beam with past history. Appl. Math. Optim., 80(1):103-133, 2019. https://doi.org/10.1007/s00245-017-9460-y.

[21] A. Lo and N. E Tatar. Uniform stability of a laminated beam with structural memory. Qual. Theory Dyn. Syst., 15(2):517-540, 2016. https://doi.org/10.1007/s12346-015-0147-y.

[22] E. Moyer and M. Miraglia. Peridynamic solutions for Timoshenko beams. Engineering, 6(6):304-317, 2014. https://doi.org/10.4236/eng.2014.66034.

[23] M.I. Mustafa. Uniform stability for thermoelastic systems with boundary time-varying delay. J. Math. Anal. Appl., 383(2):490-498, 2011. https://doi.org/10.1016/j.jmaa.2011.05.066.

[24] M.I. Mustafa. Boundary control of laminated beams with interfacial slip. $J$. Math. Phys., 59(5):051508, 2018. https://doi.org/10.1063/1.5017923.

[25] M.I. Mustafa. Laminated Timoshenko beams with viscoelastic damping. J. Math. Anal. Appl., 466(1):619-641, 2018. https://doi.org/10.1016/j.jmaa.2018.06.016.

[26] S. Nicaise and C. Pignotti. Stability and instability results of the wave equation with a delay term in the boundary or internal feedbacks. SIAM J. Control Optim., 45(5):1561-1585, 2006. https://doi.org/10.1137/060648891.

[27] C. Pignotti. A note on stabilization of locally damped wave equations with time delay. Syst. Control Lett., 61(1):92-97, 2012. https://doi.org/10.1016/j.sysconle.2011.09.016.

[28] C.A. Raposo. Exponential stability for a structure with interfacial slip and frictional damping. Appl. Math. Lett., 53:85-91, 2016. https://doi.org/10.1016/j.aml.2015.10.005.

[29] B. Said-Houari and Y. Laskri. A stability result of a Timoshenko system with a delay term in the internal feedback. Appl. Math. Comput., 217(6):2857-2869, 2010. https://doi.org/10.1016/j.amc.2010.08.021.

[30] L. Seghour, N.E. Tatar and A. Berkani. Stability of a thermoelastic laminated system subject to a neutral delay. Math. Methods Appl. Sci., 43(1):281-304, 2020. https://doi.org/10.1002/mma.5878.

[31] N.E. Tatar. Stabilization of a laminated beam with interfacial slip by boundary controls. Bound. Value Probl., 2015(1):169, 2015. https://doi.org/10.1186/s13661-015-0432-3.

[32] J.M. Wang, G.Q. Xu and S.P Yung. Exponential stabilization of laminated beams with structural damping and boundary feedback controls. SIAM J. Control Optim., 44(5):1575-1597, 2005. https://doi.org/10.1137/040610003.

[33] G.Q. Xu, S.P. Yung and L.K. Li. Stabilization of wave systems with input delay in the boundary control. ESAIM: COCV, 12(4):770-785, 2006. https://doi.org/10.1051/cocv:2006021.

[34] E. Zuazua. Uniform stabilization of the wave equation by nonlinear boundary feedback. SIAM J. Control Optim., 28(2):466-477, 1990. https://doi.org/10.1137/0328025. 